

\section{Motors of influenza vaccination uptake and vaccination advocacy in healthcare workers: a comparative study in six European countries}

George Kassianos $\mathrm{MD}^{\mathrm{a}}$, Ernest Kuchar $\mathrm{MD}^{\mathrm{b}}$, Aneta Nitsch-Osuch $\mathrm{PhD}^{\mathrm{b}}$, Jan Kyncl $\mathrm{PhD}^{\mathrm{c}}$, Andrey Galev $\mathrm{PhD}^{\mathrm{d}}$, Isme Humolli $\mathrm{MD}^{\mathrm{e}}$, Oana Falup-Pecurariu $\mathrm{MD}^{\mathrm{f}}$, Angus Thomson $\mathrm{PhD}^{\mathrm{g}}$, Christina Klein MSc ${ }^{\mathrm{g}}$, Gaëlle ValléeTourangeau $\mathrm{PhD}^{\mathrm{h}}$

${ }^{a}$ Royal College of General Practitioners, United Kingdom; ${ }^{b}$ Medical University of Warsaw, Warsaw, Poland; ${ }^{c}$ National Institute of Public Health, Prague, Czech Republic; ${ }^{\mathrm{d}}$ Military Medical Academy, Sofia, Bulgaria; ${ }^{\mathrm{e}}$ National Institute of Public Health, Prishtina, Kosovo; ${ }^{\mathrm{f}}$ Children's Clinic Hospital, Faculty of Medicine, Transilvania University, Brasov, Romania; ${ }^{\mathrm{g}}$ Sanofi Pasteur, Lyon, France; ${ }^{\mathrm{h}}$ Kingston University, London, United Kingdom

Corresponding author:

Prof Gaëlle Vallée-Tourangeau

Department of Management

Faculty of Business and Law

Kingston University

Kingston Hill

Kingston-upon-Thames

London, UK

Tel: +44(0)208 4177489

Email: G.Vallee-Tourangeau@kingston.ac.uk

Source of financial support: This study was supported by Sanofi Pasteur, Lyon France

Journal; format; counts: Vaccine; Research Article; abstract 300 words (max 300 words); body 3056 words ( $\max 3000$ words); 39 refs (max 50); 4 figures, 2 tables ( $\max 6)$ and 1 supplementary document.

Data presented in part (Czech Republic) at Options IX for the Control of Influenza, Chicago, USA 24-28 August 2016. 
ABSTRACT (300 words [300 word limit])

Background: Annual vaccination is the most effective way to prevent and control the health and economic burden caused by seasonal influenza. Healthcare workers (HCWs) play a crucial role in vaccine acceptance and advocacy for their patients. This study explored the drivers of HCWs' vaccine acceptance and advocacy in six European countries.

Methods: Healthcare workers (mainly general practitioners, specialist physicians, and nurses) voluntarily completed a questionnaire in Bulgaria $(N=485)$, Czech Republic $(N=518)$, Kosovo $(N=466)$, Poland $(N=772)$, Romania $(N=155)$, and the United Kingdom $(N=80)$. Twelve-item scales were used to analyse sentiment clusters for influenza vaccination acceptance and engagement with vaccination advocacy. Past vaccination behaviour and patient recommendation were also evaluated. All data were included in a single analysis.

Results: For vaccination acceptance, the main cluster (engaged sentiment: 68\%) showed strong positive attitudes for influenza vaccination. A second cluster (hesitant sentiment: $32 \%$ ) showed more neutral attitudes. Cluster membership was predicted by country of origin and age. The odds ratio for past vaccination in the engaged cluster was 39.6 (95\% CI 12.21-128.56) although this varied between countries. For vaccination advocacy, the main cluster (confident sentiment: $73 \%$ ) showed strong positive attitudes towards advocacy; a second cluster (diffident sentiment: $27 \%$ ) showed neutral attitudes. Cluster membership was predicted by country of origin, age and profession, with specialist physicians being the least likely to belong to the confident sentiment cluster. HCWs characterised by confident advocacy sentiments were also more likely recommend flu vaccination. Again, this association was moderated by country of origin.

Conclusions: These data show that there is room to improve both vaccination acceptance and advocacy rates in European HCWs, which would be expected to lead to higher rates of HCW vaccination. Benefits that could be expected from such an outcome are improved advocacy and better control of morbidity and mortality related to seasonal influenza infection.

Key words (max 6 key words): healthcare worker; influenza; vaccine; motivation; acceptance; advocacy

\section{INTRODUCTION}

In the European Union approximately 25-100 million individuals are infected by the seasonal influenza virus each year [1] and approximately 180 million individuals are at risk of serious complications if infected [13]. Vaccination is widely accepted by infectious disease specialists as being the most effective means of preventing seasonal influenza infection. The World Health Organisation (WHO) recommends annual vaccination for high risk groups as well as for healthcare workers (HCWs) [4]. However, specific recommendations and coverage rates may vary widely between countries in the EU [5-7].

Healthcare workers, particularly General Practitioners (GPs) and nurses, play a crucial role in vaccination decisions not only for themselves but also for their patients [8-10]. Recently there has been increasing awareness of hesitancy in the wider population towards vaccination in general and in seasonal influenza vaccination in particular, including by some HCWs [8,10-16]. People's willingness to engage in any activity is driven by both external motivations (what is required of them) and autonomous motivations (what they feel empowered to do). Previous studies have demonstrated the suitability of using questionnaires to evaluate HCW attitudes to seasonal influenza vaccination [17] as well as to a range of other infectious diseases 
(e.g., measles, pertussis) [18]. Similarly, questionnaires have been used to predict seasonal influenza vaccination rates among HCWs.

The present study departs from the traditional cognitive approach of behaviour change models such as the Health Belief Model (HBM, [19]) or the Theory of Planned Behavior (TPB, [20]), which conceptualise vaccination uptake as a deliberate choice informed by a balanced consideration of cost and benefits where the decision-maker ask herself whether she needs vaccination. Instead, our theoretical framework seeks to better understand why HCWs may want to get vaccinated, and focuses instead on motivational factors driving behaviours [20]. The willingness of an individual to engage in any activity is driven by both external motivations (what is required from the individual) and autonomous motivations (based on the individual's own assessment of the activity). Building upon the Cognitive Model of Empowerment [21], we conceive of the willingness of an individual to engage in vaccination uptake and vaccination advocacy without external pressure as determined by four personal assessments: the value/importance of the act, its impact/effectiveness, HCWs' feeling of autonomy/choice regarding the activity, and their knowledge of the activity. The aim of this study was to gauge HCWs level of engagement with influenza vaccination and vaccination advocacy and to assess whether engagement contributed to seasonal influenza vaccination uptake as well as advocacy behaviour across countries. To achieve this, we used two recently developed scales [22] and collected data from HCWs in 6 European countries, combined into a single analysis.

\section{MATERIALS AND METHODS}

\section{Participants}

Participants were HCWs from 6 European countries (Bulgaria, Czech Republic, Kosovo, Poland, Romania, and the United Kingdom). Participants were recruited via opportunity sampling with a minimum overall target of $250 \mathrm{HCW}$ ser professional category (general practitioner, specialist physician, or nurse).

\section{Design and procedure}

Data collection took place between October 2014 and December 2015. A total of 2541 participants voluntarily completed the survey in either via an online questionnaire or a paper-based version of the questionnaire. The data were screened for outliers on both the MoVac-flu and the MovAd scales. Cases with missing values $(n=18)$ or flagged as multivariate outliers based on Malahanobis distances $(n=47, p<0.001)$ were 
excluded from the analysis as this is an indication of careless responding [23]. The final sample included data from 2476 respondents. Bulgarian participants were recruited using paper questionnaires distributed at GP and preventative medicine conferences; Czech Republic participants were recruited using paper questionnaires distributed at seminars for GPs and inserted with a pre-paid return envelope in a magazine (Practicus) that is distributed to all GPs; Kosovan and Polish participants were recruited either online or using paper questionnaires; Romanian participants were recruited using paper questionnaires; UK participants were recruited via a HCWs' study day in London.

\section{Measures}

\section{Motors of influenza vaccination acceptance: MoVac-flu}

The 12-item MoVac-flu scale [22] measured the following sentiments: the sentiment that influenza vaccination is important, the sentiment that it is impactful, the feeling of knowing how influenza vaccination works, and the sentiment of autonomy regarding influenza vaccination decisions. Vaccine acceptance sentiments were measured on a 7 -point scale $(1=$ strongly disagree, $4=$ neither disagree nor agree, $7=$ strongly agree) to measure the participants' thoughts about influenza vaccination (Cohen's $\alpha=0.860$ ).

\section{Motors of engagement with vaccination advocacy: MovAd}

The 12-item MovAd scale [22] measured the following sentiments: the sentiment that vaccination advocacy is important, the sentiment that it is impactful, the feeling of knowing how to advocate vaccination, and the sentiment of autonomy regarding the decision to advocate vaccination. Vaccine advocacy sentiments were measured on the same 7-point Likert scale (Cohen's $\alpha=0.864)$.

\section{Behavioural measures}

Participants were asked whether they had received the influenza vaccine during the 2014/2015 season (autumn/winter) (immediate past behaviour). They were also asked to report how often they recommended the influenza vaccine to eligible patients (advocacy behaviour).

\section{Demographics}

Demographic data included participants' age, gender, and professional category (general practitioner, specialist physician or nurse). Table 1 summarises the demographic data. 


\section{RESULTS}

Preliminary analyses

Descriptive statistics and correlations for the MoVac-flu and MovAd scales are reported in Table 2. Normality assumptions were met as most kurtosis and skewness scores were below the upper threshold of 3.29 for large samples [24]. The only exception was the MoVac-flu item 4.1 of the Autonomy dimension (kurtosis $=$ 4.11). This deviation was corrected by using power transformation $(\lambda=2)$.

\section{Motors of flu vaccination acceptance: MoVac-flu}

Responses to the MoVac-flu scale were analysed using the Two-Step Cluster procedure from IBM SPSS 23.0, with 7 inputs: Importance (items 1, 2, 3), Impact (items 1, 2, 3), Feeling of Knowledge (items 1, 3), Depth of Knowledge (item 2), Choice (Autonomy item 1), Extrinsic Pressure (Autonomy item 2), Intrinsic Motivation (Autonomy item 3). To minimise order effects, cases were randomly ordered and cluster solutions were replicated using cases sorted in a different random order to confirm their stability.

A first solution identified two clusters with an average silhouette measure of cohesion and separation of 0.30, suggesting a "fair" clustering solution [25]. Items 1 and 2 of the Autonomy subscale as well as item 2 of the Feeling of Knowledge subscale were of weaker predictor importance (PIs $<0.15)$. The analysis was repeated without these predictors. The new and final solution identified two clusters. It was stable and showcased the average silhouette measure of cohesion and separation raised to 0.60 , suggesting a "good" clustering solution [25]. This final auto-clustering solution is presented in Table S1 in the Supplementary Results. Figure 1 presents the mean agreement ratings for each predictor as a function of cluster membership as well as the distribution of respondents across countries. The first sentiment cluster profile is the largest $(N=1675,68 \%)$. It is characterised by a strong sense that the influenza vaccine is important and impactful, a strong feeling of knowledge regarding the vaccine, and a strong sense of autonomy. This sentiment profile was labelled "engaged". By contrast, the second sentiment cluster is characterised by a neutral, slightly negative, view of the importance of the influenza vaccine and a mitigated view of its impact. This sentiment cluster is also associated with weak feelings of knowledge and no clear sense of autonomy (intrinsic motivation). It was labelled "hesitant". A one-way multivariate analysis of variance (MANOVA) confirmed all four dimensions of sentiments towards the influenza vaccination were highly differentiated between clusters,

$F(4,2471)=1445.2$, Wilk's $\Lambda=0.30, p<.001, \eta_{\mathrm{p}}{ }^{2}=0.70$ (see Table S2). 
Next, we examined whether demographic statistics were predictive of sentiments. We conducted a binary logistic regression with membership to the engaged sentiment cluster as a discrete outcome (1=belong to engaged cluster, $0=$ belong to hesitant cluster) and with country of origin, age, gender, and profession as indicators (see Tables S3 and S4 for a complete summary). Country of origin was a significant predictor of membership, Wald $\chi^{2}(5)=67.81, p<0.001$. All countries were significantly less likely to be represented in the "engaged" cluster compared to Bulgarian respondents, who were the most likely to be characterised by this sentiment. Notably, most Romanian respondents were characterised by a hesitant sentiment. They were 7.81 times less likely to be characterised by an "engaged" sentiment compared to the Bulgarian respondents. Age was the only other significant predictor of cluster membership. Older respondents more likely to belong to the engaged cluster than younger ones, with a $4 \%$ increase in the odds of being in the "engaged" cluster for every year increase in age, $\mathrm{OR}=1.04,95 \%$ CI $[1.03,1.05], B=.04, S E=0.004$, Wald $\chi^{2}(1)=65.9, \mathrm{p}<0.001$.

Next, we examined whether membership to the "engaged" sentiment cluster was a predictor of past vaccination behaviour against the flu, and whether this varied in each country. Figure 2 plots the percentage of HCWs reporting being vaccinated against the flu as a function of sentiment cluster (engaged vs. hesitant) and country of origin. We conducted a binary logistic regression with past vaccination against the flu as a discrete outcome $(1=$ vaccinated, $0=$ not vaccinated $)$ and with sentiment cluster, and sentiment cluster $\times$ country as indicators. Overall, respondents characterised by the engaged sentiment towards flu vaccination were 39.6 times more likely to have been vaccinated in the past compared to those characterised by the hesitant sentiment, $95 \%$ CI $[12.21,128.56], B=3.68, S E=0.60$, Wald $\chi^{2}(1)=37.5, p<0.001$. The odds of vaccination in the "engaged" sentiment cluster varied across countries, Wald $\chi^{2}(5)=71.0, p<0.001$. Specifically, whereas the probability of vaccination was higher for all respondents in the "engaged" sentiment cluster, this was less pronounced for Bulgarian respondents, $\mathrm{OR}=0.15,95 \% \mathrm{CI}[0.047,0.501], B=-1.88, S E=0.61$, Wald $\chi^{2}(1)=9.6, p=0.002$, Romanian, OR=0.11, 95\% CI $[0.03,0.42], B=-2.20, S E=0.68$, Wald $\chi^{2}(1)=10.5,=0.001$, and Kosovan respondents, $\mathrm{OR}=0.07,95 \% \mathrm{CI}[0.02,0.22], B=-2.72, S E=0.61$, Wald $\chi^{2}(1)=20.1, p<0.001$ (See Tables S5 and S6 for a complete results summary).

\section{Motors of vaccination advocacy: MovAd}

Responses to the MovAd scale were analysed using the same cluster procedure with 6 inputs: Importance (items 1, 2, 3), Impact (items 1, 2, 3), Feeling of Knowledge (items 1, 2, 3), Choice (Autonomy item 1), Extrinsic Pressure (Autonomy item 2), Obligation (Autonomy item 3). A first solution identified two clusters 
with an average silhouette measure of cohesion and separation of 0.40 , suggesting a "fair" clustering solution (24). Items 1, 2, and 3 of the Autonomy subscale were of weak predictor importance (PIs $<0.15)$. We re-ran the analysis without these predictors. The new and final solution identified two clusters. It was stable and showcased the average silhouette measure of cohesion and separation raised to 0.60 , suggesting a "good" clustering solution [25]. This final auto-clustering solution is presented in Table S7 from the Supplementary Results. Figure 3 presents the mean agreement ratings for each predictor as a function of cluster membership as well as the distribution of respondents across countries. The first sentiment cluster profile is the largest $(N=$ $1800,73 \%$ of respondents) and is characterised by a strong sense that vaccine advocacy is important and effective as well as a strong feeling of knowledge how to advocate vaccination. This advocacy sentiment profile was labelled "confident". By contrast, the second sentiment cluster is characterised by a neutral view of the importance of advocacy and its effectiveness, as well as a much lower feeling of knowing how to advocate vaccination. This sentiment was labelled “diffident”. A one-way MANOVA confirmed a significant differentiation between the three clusters on all dimensions, $F(3,2472)=1790.1$, Wilk's $\Lambda=0.32, p<0.001$, $\eta_{\mathrm{p}}{ }^{2}=0.69($ see Table S8).

Next we examined whether demographic statistics were predictive of advocacy sentiments. We conducted a binary logistic regression with membership to the confident sentiment cluster as a discrete outcome ( $1=$ belong to confident cluster, $0=$ belong to diffident cluster) and with country of origin, age, gender, and profession as indicators (see Tables S9 and S10 for a complete summary). Country of origin was a significant predictor of membership, Wald $\chi^{2}(5)=250.9, p<0.001$. Respondents from Kosovo and Bulgaria were the most likely to be characterised by the confident sentiment towards advocacy. All other countries were significantly less likely to be represented in the confident cluster. Notably, most Romanian respondents were characterised by a diffident sentiment and they were 14.71 times less likely to be characterised by a confident sentiment towards advocacy compared to the Bulgarian respondents. Age also had a positive effect on the odds of belonging to the confident advocate cluster, with a $4 \%$ increase for every year increase in age, OR $=1.04,95 \%$ CI $[1.03,1.05]$, $B=0.04, S E=0.01$, Wald $\chi^{2}(1)=62.3, p<0.001$. The job of respondents also made a difference, Wald $\chi^{2}(3)=10.7$, $p=0.014$. General practitioners, nurses and other healthcare workers were significantly more likely to belong to the confident sentiment cluster compared to specialist physician (ORs=1.66, 1.67, and 2.23 respectively). 
Finally, we examined whether membership to the "confident" sentiment cluster was a predictor of the frequency with which HCWs recommended flu vaccination ${ }^{1}$, and whether this varied in each country using a Univariate Analysis of Variance (ANOVA) with the advocacy behaviour as a dependent variable and cluster membership and country of origin as independent variables. Figure 4 presents the mean values for the probability of recommending the flu vaccine $(0=$ never, $100 \%=$ always $)$ as a function of cluster membership (confident vs. diffident) and respondents' country of origin. Overall, respondents characterised by the confident sentiment towards advocacy were more likely to recommend flu vaccination than those in the diffident cluster, $M_{\text {confident }}=78.4 \%, S D=17.8, M_{\text {diffident }}=59.3 \%, S D=26.0, F(1,2464)=204.61, p<.001, \eta_{\mathrm{p}}^{2}=0.08$. Countries also differed in how often they recommended the flu vaccination, $F(5,2464)=28.3, p<0.001, \eta_{\mathrm{p}}^{2}=0.05$ and the effect of advocacy cluster membership varied between countries, $F(4,2296)=7.55, \eta_{\mathrm{p}}^{2}=0.02$.

\footnotetext{
1 Advocacy behaviour was measured by asking HCWs how frequently they recommended the flu vaccine except for respondents in the Romanian survey who were asked to rate whether they "encouraged their patients to make flu vaccine" on a 7-point Likert scale. Their answers were recoded as a degree of ascent from 0 to $100 \%$ to enable a comparison with responses from other countries.
} 


\section{DISCUSSION}

Traditional theoretical frameworks conceptualise decision-making as the process of deciding whether one needs vaccination based on an evaluation of its potential benefits and associated costs. By contrast, we conceived of decisions to get vaccinated as a process driven by a consideration of whether one wants to be vaccinated based on an evaluation of the four dimensions of motivated behaviour identified by the CME [21]: the feeling of value, impact, knowledge, and autonomy. As such, our theoretical approach is better aligned more recent theoretical developments putting self-regulation at the centre stage [26,27].

A high level of HCW's autonomous motivation towards vaccination and recommending vaccination is vital for high and sustained levels of influenza vaccination coverage, which varies across Europe and in some regions has waned in recent years [16]. Good vaccination coverage is essential to reduce the significant health and economic burden associated with influenza infection [4]. In this survey of HCW sentiments in Bulgaria, the Czech Republic, Kosovo, Poland, Romania, and the UK, vaccine acceptance was generally good in each country, with only a minority of HCWs in each country being characterised by a hesitant sentiment towards influenza vaccination. Similarly, the sentiment results for advocacy were generally encouraging, with a large majority of HCWs associated with a confident sentiment towards vaccination advocacy in all countries except for Poland and Romania.

Our analyses uncovered two sentiment clusters, with most HCWs characterised by an 'engaged sentiment' which was significantly associated with past vaccination behaviour in all countries. This was especially apparent in the Czech Republic, Poland and UK samples where over $75 \%$ of HCWs identified as having an engaged sentiment towards influenza vaccination had been vaccinated against influenza in the past. For vaccination advocacy, HCWs characterised by a confident sentiment were significantly more likely to recommend influenza vaccination than those in the diffident cluster.

Vaccine hesitancy is complex with diverse root causes that can change over time, for which there are currently very few effective interventions [28]. A recommendation from a $\mathrm{HCW}$ for influenza vaccination is a strong predictor of vaccine uptake [29-32]. However providers themselves may be hesitant to vaccinate themselves, may underestimate the influence of their recommendation, and may consider themselves not to be effective in managing a vaccine-related discussion [33]. Hesitancy may not be simply overcome by providing more information or even enhancing risk perception [34]. A recent trial of a physician-targeted communication 
intervention showed no effective increase in physician self-efficacy or decrease in parental hesitancy [35].

These examples highlight the difficulty of designing interventions to overcome vaccine hesitancy. The MoVacflu and MovAd scales facilitated the definition of meaningful, and potentially actionable clusters among HCWs. This segmentation by attitudes to both vaccination and vaccination advocacy may facilitate the tailoring of interventions or communications according to the underlying motivations and current behaviours of each segment [36]. Table S11 provides illustrative guidelines for developing an effective intervention aiming to increase vaccine confidence via the implementation of the "Listen-Understand-Engage" cycle [37] and the use of the MoVac and/or MovAd scales [22]. Future research may examine whether increased line manager vaccine acceptance and advocacy can be expected to positively affect that of HCWs generally [38]. Increased knowledge is also important for full engagement [39] so increasing HCWs' sentiment of knowing could positively affect their sentiments towards influenza vaccination.

Limitations of this study include the sampling strategy: all samples were recruited through convenience, or opportunistic sampling and thus cannot be considered as representative of the healthcare populations in the respective countries. It is also possible that the voluntary nature of participation in this survey may have skewed the data in favour of acceptance and advocacy and it is assumed that those holding very negative views may have declined to take part. The validity of the generalisation of these data to other European countries, or globally, is also untested, and the range of healthcare context and vaccine cost in the different countries was not accounted for.

Nevertheless, the MoVac-flu and MovAd surveys provided an effective tool to monitor, understand, and compare the proportions of HCWs who feel engaged regarding flu vaccination and feel confident in advocating the vaccine. To our knowledge this is one of the first attempts to measure self-perceived efficacy in advocating vaccination. These tools could be used to design and measure outcomes of $\mathrm{HCW}$-focused interventions that aim to increase provider confidence in influenza vaccination and in recommending influenza vaccination. 


\section{ACKNOWLEDGEMENTS}

The authors thank all HCWs who participated in this study.

The authors also thank the study staff in each country for distributing and collecting the questionnaires, under the supervision of Dr Galev (Bulgaria), Dr Kyncl (Czech Republic), Dr Humoli (Kosovo), Drs Nitsch-Osuch and Kuchar (Poland), Dr Falup-Pecurariu (Romania), and Dr Kassianos (UK). The data were analysed and summarised by Professor Gaëlle Vallée-Tourangeau. This manuscript was prepared with the assistance of a professional medical writer, Dr Andrew Lane (Lane Medical Writing), funded by Sanofi Pasteur, in accordance with the European Medical Writers Association guidelines and Good Publication Practice.

This study was funded by Sanofi Pasteur. 


\section{CONFLICTS OF INTEREST}

AT and CK are employees of Sanofi Pasteur. GV-T received funding from Sanofi Pasteur to design, and supervise the data collection for this study.

GK (UK), EK (Poland), AN-O (Poland), JK (Czech Republic), AG (Bulgaria), IH (Kosovo), and OF-P (Romania) have no relevant conflicts of interest. 


\section{REFERENCES}

[1] British Global and Travel Health Association, Kassianos G. Willingness of European healthcare workers to undergo vaccination against seasonal influenza: current situation and suggestions for improvement. Drugs Context 2015;4:1-9. doi:10.7573/dic.212268.

[2] Factsheet about seasonal influenza. Eur Cent Dis Prev Control ECDC n.d. https://ecdc.europa.eu/en/seasonal-influenza/facts/factsheet (accessed December 5, 2017).

[3] Preaud E, Durand L, Macabeo B, Farkas N, Sloesen B, Palache A, et al. Annual public health and economic benefits of seasonal influenza vaccination: a European estimate. BMC Public Health 2014;14:813. doi:10.1186/1471-2458-14-813.

[4] WHO | Influenza (Seasonal). WHO n.d. http://www.who.int/mediacentre/factsheets/fs211/en/ (accessed December 5, 2017).

[5] European Commission. State of play on implementation of the Council Recommendation of 22 December 2009 on seasonal influenza vaccination (2009/1019/EU). Brussels: European Commission; 2014.

[6] Blank PR, Schwenkglenks M, Szucs TD. Vaccination coverage rates in eleven European countries during two consecutive influenza seasons. J Infect 2009;58:446-58. doi:10.1016/j.jinf.2009.04.001.

[7] Commission of the European Communities. Proposal for a council recommendation on seasonal influenza vaccination. Brussels: 2009.

[8] Verger P, Fressard L, Collange F, Gautier A, Jestin C, Launay O, et al. Vaccine Hesitancy Among General Practitioners and Its Determinants During Controversies: A National Cross-sectional Survey in France. EBioMedicine 2015;2:891-7. doi:10.1016/j.ebiom.2015.06.018.

[9] Benin AL. Qualitative Analysis of Mothers' Decision-Making About Vaccines for Infants: The Importance of Trust. PEDIATRICS 2006;117:1532-41. doi:10.1542/peds.2005-1728.

[10] Dubé E, Laberge C, Guay M, Bramadat P, Roy R, Bettinger JA. Vaccine hesitancy: An overview. Hum Vaccines Immunother 2013;9:1763-73. doi:10.4161/hv.24657.

[11] Freed GL, Clark SJ, Cowan AE, Coleman MS. Primary care physician perspectives on providing adult vaccines. Vaccine 2011;29:1850-4. doi:10.1016/j.vaccine.2010.12.097.

[12] Gust DA, Darling N, Kennedy A, Schwartz B. Parents With Doubts About Vaccines: Which Vaccines and Reasons Why. PEDIATRICS 2008;122:718-25. doi:10.1542/peds.2007-0538.

[13] Rubin GJ, Potts HWW, Michie S. Likely uptake of swine and seasonal flu vaccines among healthcare workers. A cross-sectional analysis of UK telephone survey data. Vaccine 2011;29:2421-8.

doi:10.1016/j.vaccine.2011.01.035.

[14] Schwarzinger M, Verger P, Guerville M-A, Aubry C, Rolland S, Obadia Y, et al. Positive attitudes of French general practitioners towards $\mathrm{A} / \mathrm{H} 1 \mathrm{~N} 1$ influenza-pandemic vaccination: A missed opportunity to increase vaccination uptakes in the general public? Vaccine 2010;28:2743-8.

doi:10.1016/j.vaccine.2010.01.027.

[15] Pulcini C, Massin S, Launay O, Verger P. Knowledge, attitudes, beliefs and practices of general practitioners towards measles and MMR vaccination in southeastern France in 2012. Clin Microbiol Infect 2014;20:38-43. doi:10.1111/1469-0691.12194.

[16] Karafillakis E, Dinca I, Apfel F, Cecconi S, Würz A, Takacs J, et al. Vaccine hesitancy among healthcare workers in Europe: A qualitative study. Vaccine 2016;34:5013-20.

doi:10.1016/j.vaccine.2016.08.029.

[17] Real K, Kim S, Conigliaro J. Using a validated health promotion tool to improve patient safety and increase health care personnel influenza vaccination rates. Am J Infect Control 2013;41:691-6.

doi:10.1016/j.ajic.2012.09.027.

[18] Martinez L, Fofana F, Raineri F, Arnould P, Benmedjahed K, Coindard G, et al. Scoring and psychometric validation of the 'Determinants of Intentions to Vaccinate' (DIVAC) questionnaire. BMC Fam Pract 2016;17. doi:10.1186/s12875-016-0539-3.

[19] Becker MH, Maiman LA. Sociobehavioral determinants of compliance with health and medical care recommendations. Med Care 1975;13:10-24.

[20] Maiman LA, Becker MH. The Health Belief Model: Origins and Correlates in Psychological Theory. Health Educ Monogr 1974;2:336-53. doi:10.1177/109019817400200404.

[21] Thomas KW, Velthouse BA. Cognitive elements of empowerment: An" interpretive" model of intrinsic task motivation. Acad Manage Rev 1990;15:666-681.

[22] Vallée-Tourangeau G, Promberger M, Moon K, Wheelock A, Sirota M, Norton C, et al. Motors of influenza vaccination uptake and vaccination advocacy in healthcare workers: Development and validation of two short scales. Vaccine 2017. doi:10.1016/j.vaccine.2017.08.025.

[23] Meade AW, Craig SB. Identifying careless responses in survey data. Psychol Methods 2012;17:43755. doi:10.1037/a0028085. 
[24] Field AP. Discovering statistics using SPSS. 3rd ed. Los Angeles: SAGE Publications; 2009.

[25] Kaufman L, Rousseeuw PJ. Finding Groups in Data: an Introduction to Cluster Analysis. Hoboken: John Wiley \& Sons, Inc.; 2009.

[26] Ryan RM, Deci EL. Self-determination theory and the facilitation of intrinsic motivation, social development, and well-being. Am Psychol 2000;55:68.

[27] Michie S, Van Stralen MM, West R. The behaviour change wheel: a new method for characterising and designing behaviour change interventions. Implement Sci 2011;6:42.

[28] Dubé E, Gagnon D, MacDonald NE. Strategies intended to address vaccine hesitancy: Review of published reviews. Vaccine 2015;33:4191-203. doi:10.1016/j.vaccine.2015.04.041.

[29] Gust D., Woodruff R, Kennedy A, Brown C, Sheedy K, Hibbs B. Parental perceptions surrounding risks and benefits of immunization. Semin Pediatr Infect Dis 2003;14:207-12. doi:10.1016/S1045-

1870(03)00035-9.

[30] Opel DJ, Heritage J, Taylor JA, Mangione-Smith R, Salas HS, DeVere V, et al. The Architecture of Provider-Parent Vaccine Discussions at Health Supervision Visits. PEDIATRICS 2013;132:1037-46.

doi:10.1542/peds.2013-2037.

[31] Wheelock A, Thomson A, Sevdalis N. Social and psychological factors underlying adult vaccination behavior: lessons from seasonal influenza vaccination in the US and the UK. Expert Rev Vaccines 2013;12:893-901. doi:10.1586/14760584.2013.814841.

[32] Williams SE, Rothman RL, Offit PA, Schaffner W, Sullivan M, Edwards KM. A Randomized Trial to Increase Acceptance of Childhood Vaccines by Vaccine-Hesitant Parents: A Pilot Study. Acad Pediatr 2013;13:475-80. doi:10.1016/j.acap.2013.03.011.

[33] Wallace C. Effects of a web based decision aid on parental attitudes to MMR vaccination: a before and after study. BMJ 2006;332:146-9. doi:10.1136/bmj.38678.681840.68.

[34] Llupià A, Mena G, Olivé V, Quesada S, Aldea M, Sequera VG, et al. Evaluating influenza vaccination campaigns beyond coverage: A before-after study among health care workers. Am J Infect Control 2013;41:674-8. doi:10.1016/j.ajic.2013.04.006.

[35] Henrikson NB, Opel DJ, Grothaus L, Nelson J, Scrol A, Dunn J, et al. Physician Communication Training and Parental Vaccine Hesitancy: A Randomized Trial. PEDIATRICS 2015;136:70-9. doi:10.1542/peds.2014-3199.

[36] Nowak GJ, Gellin BG, MacDonald NE, Butler R. Addressing vaccine hesitancy: The potential value of commercial and social marketing principles and practices. Vaccine 2015;33:4204-11.

doi:10.1016/j.vaccine.2015.04.039.

[37] Thomson A, Watson M. Listen, Understand, Engage. Sci Transl Med 2012;4:138ed6-138ed6. doi:10.1126/scitranslmed.3004264.

[38] Corace K, Prematunge C, McCarthy A, Nair RC, Roth V, Hayes T, et al. Predicting influenza vaccination uptake among health care workers: What are the key motivators? Am J Infect Control 2013;41:679_ 84. doi:10.1016/j.ajic.2013.01.014.

[39] Centola D. The Spread of Behavior in an Online Social Network Experiment. Science 2010;329:11947. doi:10.1126/science.1185231. 
Table 1 Demographic characteristics of the samples

\begin{tabular}{|c|c|c|c|c|c|c|c|}
\hline \multirow[b]{2}{*}{ Variable } & \multicolumn{6}{|c|}{ Country } & \multirow[b]{2}{*}{ TOTAI } \\
\hline & BGR & CZE & GBR & POL & ROU & XKX & \\
\hline$N$ & 485 & 518 & 80 & 772 & 155 & 466 & 2476 \\
\hline \multicolumn{8}{|l|}{ Age } \\
\hline$M$ & 49.38 & 50.98 & 49.81 & 48.63 & 35.84 & 45.5 & 47.92 \\
\hline$S D$ & 7.47 & 12.16 & 9.78 & 11.53 & 9.39 & 9.2 & 10.98 \\
\hline Minimum & 23 & 18 & 20 & 23 & 19 & 22 & 18 \\
\hline Maximum & 70 & 81 & 67 & 83 & 62 & 63 & 83 \\
\hline $18-29$ years $(\%)$ & 3.30 & 6.58 & 5.56 & 6.74 & 37.01 & 4.29 & 7.42 \\
\hline $30-49$ years $(\%)$ & 50.93 & 35.78 & 38.89 & 52.20 & 55.19 & 64.59 & 50.65 \\
\hline $50-65$ years $(\%)$ & 45.57 & 48.94 & 54.17 & 33.68 & 7.79 & 31.12 & 37.71 \\
\hline Over 65 years (\%) & 0.21 & 8.70 & 1.39 & 7.38 & 0.00 & 0.00 & 4.22 \\
\hline \multicolumn{8}{|l|}{ Gender } \\
\hline Female $(n)$ & 393 & 382 & 77 & 516 & 117 & 304 & 1789 \\
\hline Male $(n)$ & 92 & 136 & 2 & 256 & 37 & 162 & 685 \\
\hline Missing & 0 & 0 & 1 & 0 & 1 & 0 & 2 \\
\hline \multicolumn{8}{|l|}{ Profession } \\
\hline General practitioner & 478 & 451 & 1 & 675 & 0 & 174 & 1779 \\
\hline Specialist physician & 0 & 8 & 0 & 0 & 49 & 38 & 95 \\
\hline Nurse & 2 & 13 & 76 & 84 & 73 & 224 & 472 \\
\hline Other & 5 & 46 & 3 & 13 & 33 & 30 & 130 \\
\hline
\end{tabular}


Table 2 Descriptive statistics, correlations, and coefficient alpha for MoVac-flu and MovAd sentiments

\begin{tabular}{|c|c|c|c|c|c|c|c|c|c|c|c|}
\hline \multirow[b]{2}{*}{ Sentiments } & \multirow[b]{2}{*}{$M$} & \multirow[b]{2}{*}{$S D$} & \multirow[b]{2}{*}{ Skewness } & \multirow[b]{2}{*}{ Kurtosis } & \multirow[b]{2}{*}{ alpha } & \multicolumn{6}{|c|}{ Correlations } \\
\hline & & & & & & 1 & 2 & 3 & 3.2 & 4.1 & 4.2 \\
\hline \multicolumn{12}{|c|}{ MoVac-flu sentiments } \\
\hline 1. Importance & 5.45 & 1.74 & -1.09 & 0.31 & 0.90 & & & & & & \\
\hline 2. Impact & 5.86 & 1.42 & -1.51 & 2.23 & 0.87 & $0.81^{* * *}$ & & & & & \\
\hline 3. Feeling of Knowledge & 6.06 & 1.32 & -1.74 & 0.68 & $0.86^{2}$ & $0.72^{* * *}$ & $0.79^{* * *}$ & & & & \\
\hline 3.2 Depth of Knowledge ${ }^{3}$ & 5.61 & 2.11 & -1.25 & 0.30 & & 0.02 & $0.06^{* *}$ & $0.12^{* * *}$ & & & \\
\hline 4. Autonomy & & & & & 0.16 & & & & & & \\
\hline 4.1. Choice ${ }^{1}$ & 6.23 & 1.48 & -1.64 & 1.45 & $0.08^{4}$ & $0.19^{* * *}$ & $0.23^{* * *}$ & $0.29^{* * *}$ & $0.09^{* * *}$ & & \\
\hline 4.2. Extrinsic Pressure & 3.07 & 2.27 & 0.60 & -1.19 & $0.33^{4}$ & $0.05^{*}$ & $-0.04^{*}$ & -0.01 & $-0.24^{* * *}$ & 0.02 & \\
\hline 4.3. Intrinsic Motivation & 5.74 & 1.96 & -1.46 & 0.74 & $-0.07^{4}$ & $0.048^{* * *}$ & $0.53^{* * *}$ & $0.44^{* * *}$ & $0.05^{*}$ & $0.21^{* * *}$ & $-0.04^{*}$ \\
\hline \multicolumn{12}{|c|}{ MovAd sentiments } \\
\hline 1. Importance & 5.87 & 1.30 & -1.32 & 1.30 & 0.83 & & & & & & \\
\hline 2. Impact & 5.67 & 1.20 & -1.04 & 1.02 & 0.84 & $0.67^{* * *}$ & & & & & \\
\hline 3. Feeling of Knowledge & 5.85 & 1.25 & -1.38 & 1.73 & 0.90 & $0.62^{* * *}$ & $0.54^{* * *}$ & & & & \\
\hline 4. Autonomy & & & & & 0.31 & & & & & & \\
\hline 4.1. Choice & 5.17 & 1.96 & -0.82 & -0.52 & $0.09^{4}$ & $0.25^{* * *}$ & $0.17^{* * *}$ & $0.21^{* * *}$ & & & \\
\hline 4.2. Extrinsic Pressure & 3.16 & 2.16 & 0.55 & -1.15 & $0.27^{4}$ & $0.07^{* * *}$ & $0.07^{* * *}$ & 0.03 & & $0.18^{* * *}$ & \\
\hline 4.3. Obligation & 5.85 & 1.55 & -1.44 & 1.37 & $0.30^{4}$ & $0.66^{* * *}$ & $0.51^{* * *}$ & $0.56^{* * *}$ & & $0.16^{* * *}$ & $0.05^{*}$ \\
\hline
\end{tabular}

${ }_{* *}^{*} \quad p<0.05$

** $\quad p<0.01$

${ }^{* * *} \quad p<0.001$

${ }^{1}$ Skewness, kurtosis, and correlations scores based on power-transformed variables $(\lambda=2)$.

${ }^{2}$ Cohen's alpha if item 3.2 of the Feeling of Knowledge subscale for MoVac-flu is removed.

33.2 Depth of Knowledge: "How the flu jab works to protect my health is a mystery to me" (reverse-coded).

${ }^{4}$ Cohen's alpha if the corresponding item is removed. 
Figure 1 MoVac-flu profiles for the "engaged" and "hesitant" clusters and their distributions across countries ordered from the most to the least represented in the "engaged" cluster

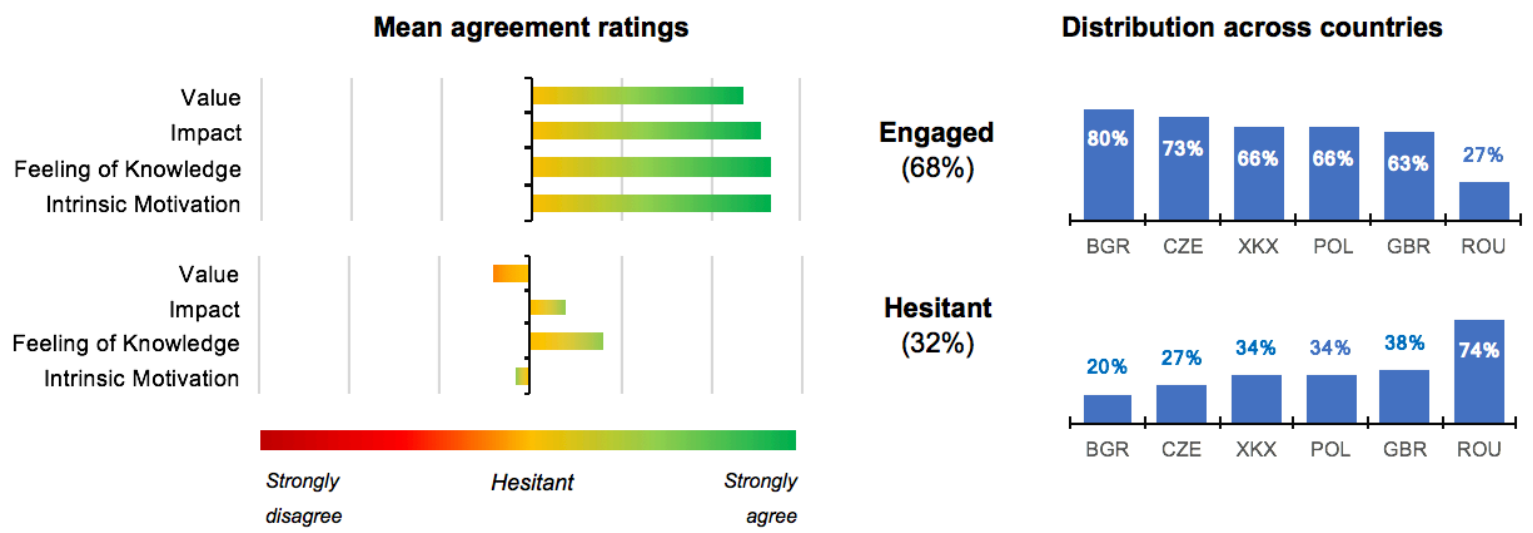


Figure 2 Percentage of healthcare workers reporting being vaccinated against influenza as a function of sentiment cluster (“engaged" vs. "hesitant" clusters) and country ordered from the smallest to the largest vaccination gap between "hesitant" and "engaged" sentiment clusters

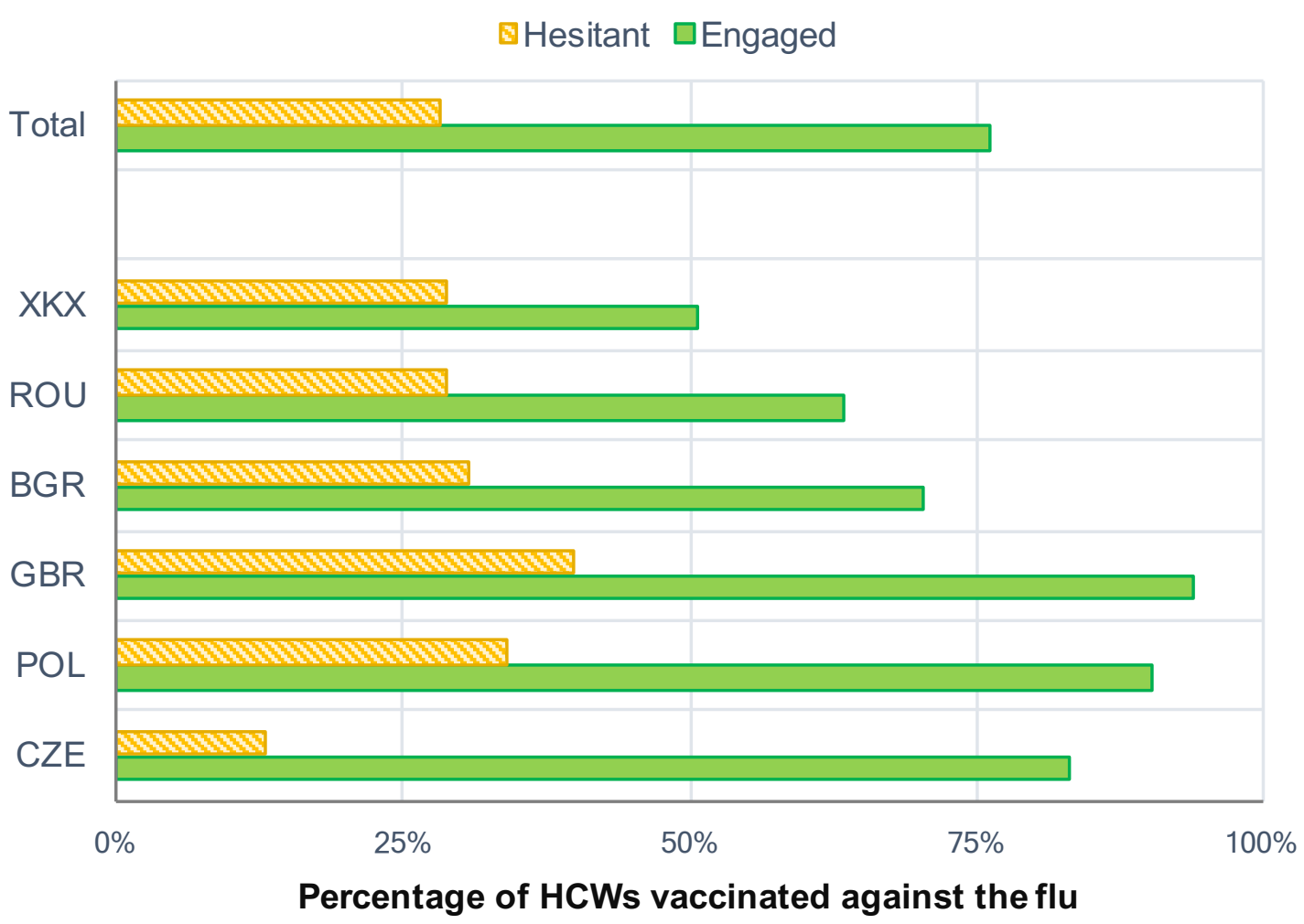


Figure 3 MovAd profiles for the "confident" and "diffident" sentiment clusters and their distributions across countries ordered from the most to the least represented in the “confident" cluster

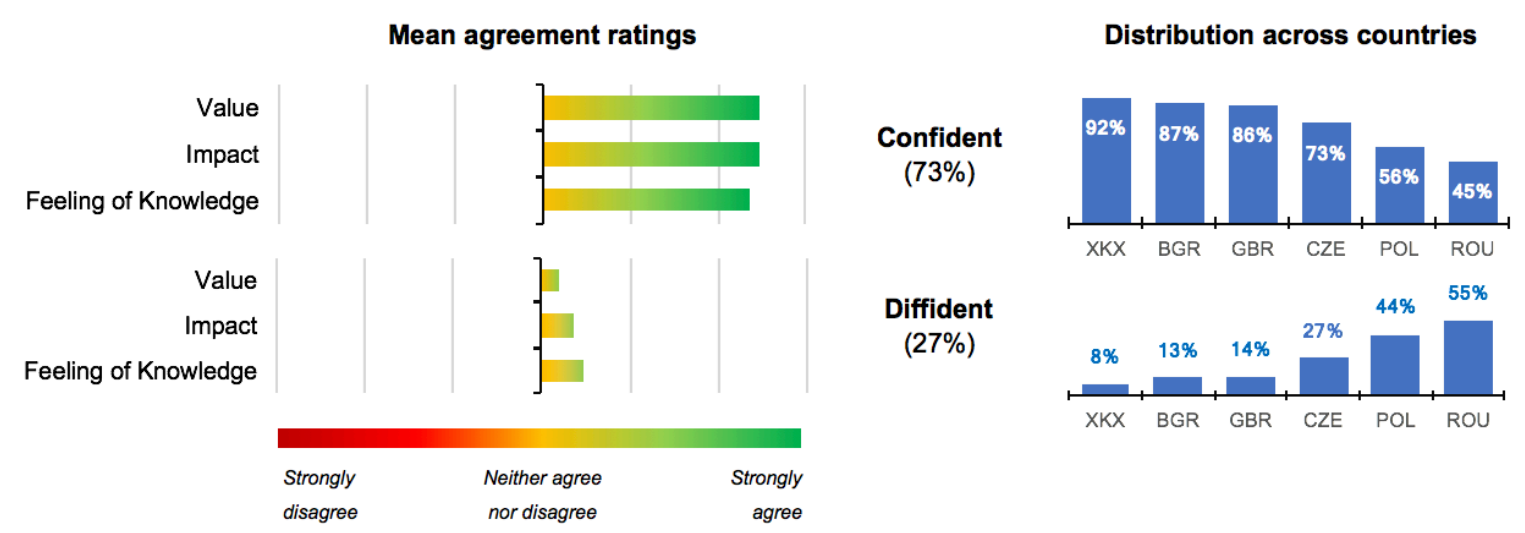


Figure 4 Mean probability that healthcare workers will recommend influenza vaccination as a function of advocacy sentiment cluster (“diffident” vs. "confident” clusters) and country ordered from the largest to the smallest gap in recommendation probability between "diffident" and "confident" sentiment clusters (100\%=always recommend, $0 \%=$ never recommend)

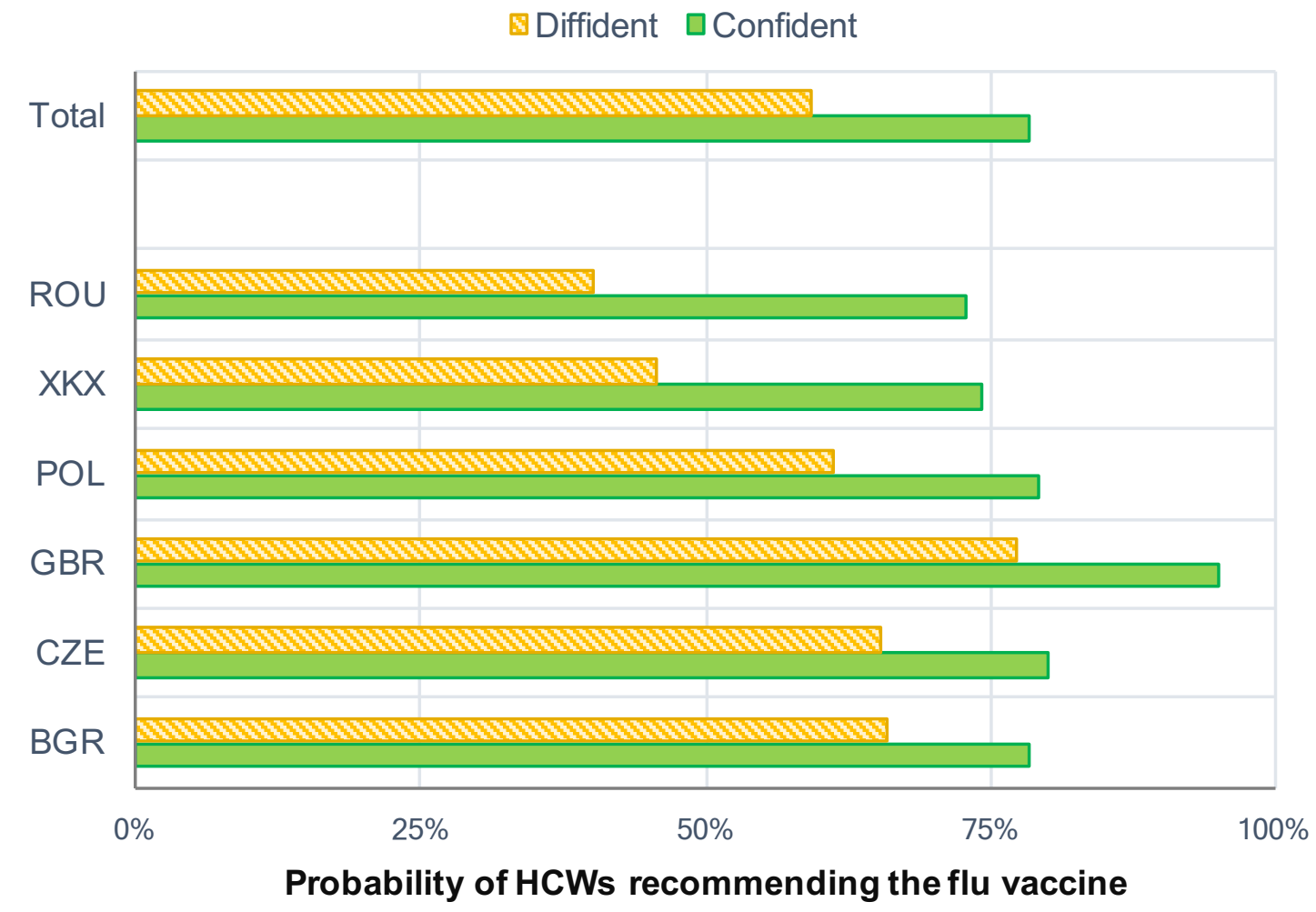


Table S1 Auto-clustering solution for the MoVac-flu scale

\begin{tabular}{|c|c|c|c|c|c|}
\hline Number of Clusters & & $\begin{array}{c}\text { Schwarz's Bayesian } \\
\text { Criterion (BIC) }\end{array}$ & $\begin{array}{c}\text { BIC } \\
\text { Change }^{a} \\
\end{array}$ & $\begin{array}{c}\text { Ratio of BIC } \\
\text { Changes }\end{array}$ & $\begin{array}{c}\text { Ratio of Distance } \\
\text { Measures }\end{array}$ \\
\hline & 1 & 6925.4 & & & \\
\hline & 2 & 3780.8 & -3144.7 & 1.00 & 5.09 \\
\hline & 3 & 3213.4 & -567.3 & 0.18 & 1.59 \\
\hline & 4 & 2880.2 & -333.2 & 0.11 & 1.22 \\
\hline & 5 & 2619.3 & -261.0 & 0.08 & 1.26 \\
\hline & 6 & 2424.0 & -195.3 & 0.06 & 1.30 \\
\hline
\end{tabular}

${ }^{a}$ The changes are from the previous number of clusters in the table.

${ }^{\mathrm{b}}$ The ratios of changes are relative to the change for the two cluster solution.

${ }^{\mathrm{c}}$ The ratios of distance measures are based on the current number of clusters against the previous number of clusters.

Table S2 Predictor importance, and one-way analysis of variance of cluster profile distinctiveness for the MoVac-flu scale

\begin{tabular}{lcccc}
\hline Predictors & PI & $F(1,2474)$ & $p$ & $\eta_{p}^{2}$ \\
\hline Value $(1,2,3)$ & 1.00 & 3087.0 & $<0.001$ & 0.56 \\
Impact $(1,2,3)$ & 1.00 & 2566.8 & $<0.001$ & 0.51 \\
Feeling of Knowledge (1,3) & 0.97 & 1822.3 & $<0.001$ & 0.42 \\
Intrinsic Motivation (3) & 1.00 & 2050.4 & $<0.001$ & 0.45 \\
\hline
\end{tabular}


Table S3 Mean values and frequencies for predictor variables as a function of cluster membership for the MoVac-flu scale

\begin{tabular}{lcccc}
\hline Predictors & Engaged & Hesitant & $\begin{array}{c}\chi^{2}(1) \text { or } \\
t(2465)\end{array}$ & $p$ \\
\hline Country (\%) & & & & \\
BGR & $79.8 \%$ & $20.2 \%$ & 172.2 & $<0.001$ \\
CZE & $73.0 \%$ & $27.0 \%$ & 109.4 & $<0.001$ \\
XKX & $66.3 \%$ & $33.7 \%$ & 49.6 & $<0.001$ \\
POL & $66.1 \%$ & $33.9 \%$ & 79.7 & $<0.001$ \\
GBR & $62.5 \%$ & $37.5 \%$ & 5.0 & 0.025 \\
ROU & $26.5 \%$ & $73.5 \%$ & 34.4 & $<0.001$ \\
Age & & & & $<0.001$ \\
$M$ & 49.7 & 44.2 & 12.0 & \\
SD & 10.4 & 11.2 & & 0.031 \\
Female (\%) & $75.1 \%$ & $71.0 \%$ & 4.7 & $<0.608$ \\
Profession & & & & $<0.001$ \\
Specialist physician & $52.6 \%$ & $47.4 \%$ & 0.3 & 0.001 \\
General practitioner & $72.1 \%$ & $27.9 \%$ & 348.2 & 12.2 \\
Nurse & $58.1 \%$ & $41.9 \%$ & 0.3 & \\
Other & $52.3 \%$ & $47.7 \%$ & & \\
\hline
\end{tabular}

Note. A t-test was used for the Age predictor variable; a Chi-square test was used for all other variables. 
Table S4 Summary of the binary logistic regression analysis predicting membership to the "engaged" sentiment cluster towards flu vaccination

\begin{tabular}{|c|c|c|c|c|c|c|c|}
\hline Predictors & $B$ & $S E$ & $O R$ & $95 \% \mathrm{CI}$ & Wald $\chi^{2}$ & df & $p$ \\
\hline Country (\%) & & & & & 67.8 & 5 & $<0.001$ \\
\hline $\mathrm{CZE}$ & -0.419 & 0.154 & 0.658 & {$[0.49,0.89]$} & 7.4 & 1 & 0.007 \\
\hline $\mathrm{XKX}$ & -0.595 & 0.178 & 0.552 & {$[0.39,0.78]$} & 11.2 & 1 & 0.001 \\
\hline POL & -0.698 & 0.140 & 0.497 & {$[0.38,0.66]$} & 24.9 & 1 & $<0.001$ \\
\hline GBR & -0.657 & 0.314 & 0.519 & {$[0.28,0.96]$} & 4.4 & 1 & 0.036 \\
\hline ROU & -2.059 & 0.272 & 0.128 & {$[0.08,0.22]$} & 57.5 & 1 & $<0.001$ \\
\hline Age & 0.036 & 0.004 & 1.037 & {$[1.03,1.05]$} & 65.9 & 1 & $<0.001$ \\
\hline Female $(\%)$ & 0.199 & 0.107 & 1.220 & {$[0.99,1.51]$} & 3.4 & 1 & 0.064 \\
\hline Profession & & & & & 1.5 & 3 & 0.680 \\
\hline General practitioner & -0.227 & 0.283 & 0.797 & {$[0.46,1.39]$} & 0.6 & 1 & 0.423 \\
\hline Nurse & -0.235 & 0.274 & 0.790 & {$[0.46,1.35]$} & 0.7 & 1 & 0.391 \\
\hline Other & -0.385 & 0.319 & 0.680 & {$[0.36,1.27]$} & 1.5 & 1 & 0.227 \\
\hline
\end{tabular}

Note. $\mathrm{CI}=$ confidence interval for odds ratio (OR). Reference category for Country=BGR. Reference category for Profession=Specialist Physician. $R^{2}=0.128$ (Nagelkerke). Model $\chi^{2}(10)=237.8, p<0.001,-2 \mathrm{LL}=2862.7$.

Table S5 Frequencies for the MoVac-flu "engaged" cluster membership as a function of country and vaccination behaviour

\begin{tabular}{lcccc}
\hline Predictors & Vaccinated & Not Vaccinated & $\chi^{2}(1)$ & $p$ \\
\hline Engaged (\%) & $84.9 \%$ & $40.9 \%$ & 522.9 & $<0.001$ \\
Engaged | GBR & $79.8 \%$ & $14.3 \%$ & 28.2 & $<0.001$ \\
Engaged | POL & $83.8 \%$ & $22.1 \%$ & 269.0 & $<0.001$ \\
Engaged | CZE & $94.6 \%$ & $34.4 \%$ & 218.8 & $<0.001$ \\
Engaged | BGR & $90.1 \%$ & $62.6 \%$ & 53.2 & $<0.001$ \\
Engaged | ROU & $44.1 \%$ & $15.6 \%$ & 15.2 & $<0.001$ \\
Engaged | XKX & $77.7 \%$ & $57.6 \%$ & 20.8 & $<0.001$ \\
\hline
\end{tabular}

Note. A Chi-square test was used for all variables. 
Table S6 Summary of the binary logistic regression analysis predicting membership to the MoVac-flu "engaged" sentiment cluster towards flu vaccination

\begin{tabular}{|c|c|c|c|c|c|c|c|}
\hline Predictors & $B$ & $S E$ & $O R$ & $95 \% \mathrm{CI}$ & Wald $\chi^{2}$ & $\mathrm{df}$ & $p$ \\
\hline Block 1 & & & & & & & \\
\hline $\begin{array}{l}\text { MoVac Sentiment } \\
\text { Block } 2\end{array}$ & 2.10 & 0.10 & 8.14 & {$[6.73,9.85]$} & 465.3 & 1 & $<0.001$ \\
\hline MoVac Sentiment & 3.68 & 0.60 & 39.615267 & {$[12.21,128.56]$} & 37.5 & 1 & $<0.001$ \\
\hline MoVac $\times$ Country & & & & & 171.0 & 5 & $<0.001$ \\
\hline POL & -0.51 & 0.61 & 0.60 & {$[0.18,2.00]$} & 0.7 & 1 & 0.406 \\
\hline CZE & -1.16 & 0.61 & 0.31 & {$[0.10,1.04]$} & 3.6 & 1 & 0.057 \\
\hline BGR & -1.88 & 0.61 & 0.15 & {$[0.047,0.501]$} & 9.6 & 1 & 0.002 \\
\hline ROU & -2.20 & 0.68 & 0.11 & {$[0.03,0.42]$} & 10.5 & 1 & 0.001 \\
\hline $\mathrm{XKX}$ & -2.72 & 0.61 & 0.07 & {$[0.02,0.22]$} & 20.1 & 1 & $<0.001$ \\
\hline
\end{tabular}

Note. $\mathrm{CI}=$ confidence interval for odds ratio (OR). Reference category for Country=GBR. Block 1: $R^{2}=0.26\left(\right.$ Nagelkerke). Model $\chi^{2}(1)=526.9, p<0.001,-2 \mathrm{LL}=2789.5$.

Block 2: $R^{2}=0.34$ (Nagelkerke). Block $\chi^{2}(5)=194.0, p<0.001$, Model $\chi^{2}(6)=720.8, p<0.001,-2 \mathrm{LL}=2595.6$.

Table S7 Auto-clustering solution for the MovAd scale

\begin{tabular}{rrrrr}
\hline Number of Clusters & Schwarz's Bayesian Criterion (BIC) & BIC Change $^{\mathrm{a}}$ & Ratio of BIC Changes $^{\mathrm{b}}$ & Ratio of Distance Measures $^{\mathrm{c}}$ \\
\hline 1 & 5194.1 & & & \\
2 & 3145.5 & -2048.6 & 1.00 & 4.02 \\
3 & 2671.5 & -474.0 & 0.23 & 1.32 \\
4 & 2322.7 & -348.8 & 0.17 & 1.95 \\
5 & 2167.2 & -155.6 & 0.08 & 1.31 \\
6 & 2059.8 & -107.3 & 0.05 & 1.28 \\
\hline
\end{tabular}

${ }^{\mathrm{a}}$ The changes are from the previous number of clusters in the table.

${ }^{\mathrm{b}}$ The ratios of changes are relative to the change for the two cluster solution.

${ }^{\mathrm{c}}$ The ratios of distance measures are based on the current number of clusters against the previous number of clusters. 
Table S8 Predictor importance, and one-way analysis of variance of cluster profile distinctiveness for the MovAd scale

\begin{tabular}{lcccc}
\hline Predictors & PI & $F(1,2474)$ & $p$ & $\eta_{p}^{2}$ \\
\hline Value $(1,2,3)$ & 1.00 & 3920.3 & $<0.001$ & 0.61 \\
Impact $(1,2,3)$ & 1.00 & 1891.1 & $<0.001$ & 0.43 \\
Knowledge $(1,2,3)$ & 1.00 & 1960.1 & $<0.001$ & 0.44 \\
\hline
\end{tabular}


Table S9 Mean values and frequencies for predictor variables as a function of cluster membership for the MovAd scale

\begin{tabular}{lcccc}
\hline Predictors & Confident & Diffident & $\begin{array}{c}\chi^{2}(1) \text { or } \\
t(2465)\end{array}$ & $p$ \\
\hline Country (\%) & & & \\
XKX & $92.7 \%$ & $7.3 \%$ & 339.9 & $<0.001$ \\
BGR & $87.6 \%$ & $12.4 \%$ & 274.7 & $<0.001$ \\
GBR & $86.3 \%$ & $13.8 \%$ & 42.1 & $<0.001$ \\
CZE & $72.4 \%$ & $27.6 \%$ & 103.9 & $<0.001$ \\
POL & $56.0 \%$ & $44.0 \%$ & 11.0 & $<0.001$ \\
ROU & $46.5 \%$ & $53.5 \%$ & 0.8 & 0.377 \\
Age & & & & $<0.001$ \\
$M$ & 49.0 & 44.9 & 8.4 & \\
SD & 10.4 & 11.9 & & $<0.066$ \\
Female (\%) & 73.3 & 69.6 & 3.4 & $<0.001$ \\
Profession & & & & $<0.001$ \\
Specialist physician & $71.6 \%$ & $28.4 \%$ & 17.7 & $<0.001$ \\
General practitioner & $71.3 \%$ & $28.7 \%$ & 323.8 & $<0.001$ \\
Nurse & $79.9 \%$ & $20.1 \%$ & 168.5 & 20.8 \\
Other & $70.0 \%$ & $30.0 \%$ & & \\
\hline
\end{tabular}

Note. $A$ t-test was used for the Age predictor variable; a Chi-square test was used for all other variables. 
Table S10 Summary of the binary logistic regression analysis predicting membership to the "confident" sentiment cluster towards vaccination advocacy

\begin{tabular}{lccccccc}
\hline Predictors & $B$ & $S E$ & $O R$ & $95 \%$ CI & Wald $\chi^{2}$ & df & $p$ \\
\hline Country (\%) & & & & & 250.9 & 5 & $<0.001$ \\
$\quad$ BGR & -0.467 & 0.247 & 0.627 & {$[0.39,1.02]$} & 3.6 & 1 & 0.059 \\
GBR & -0.864 & 0.430 & 0.421 & {$[0.18,0.98]$} & 4.0 & 1 & 0.045 \\
CZE & -1.556 & 0.223 & 0.211 & {$[0.14,0.33]$} & 48.6 & 1 & $<0.001$ \\
POL & -2.210 & 0.208 & 0.110 & {$[0.07,0.17]$} & 112.5 & 1 & $<0.001$ \\
$\quad$ ROU & -2.687 & 0.270 & 0.068 & {$[0.04,0.12]$} & 99.2 & 1 & $<0.001$ \\
Age & 0.037 & 0.005 & 1.038 & {$[1.03,1.05]$} & 62.3 & 1 & $<0.001$ \\
Female (\%) & -0.159 & 0.112 & 0.853 & {$[0.68,1.06]$} & 2.0 & 1 & 0.158 \\
Profession & & & & & 10.7 & 3 & 0.007 \\
$\quad$ General practitioner & 0.509 & 0.188 & 1.664 & {$[1.15,2.40]$} & 7.4 & 1 & 0.038 \\
$\quad$ Nurse & 0.514 & 0.247 & 1.671 & {$[1.03,2.71]$} & 4.3 & 1 & 0.019 \\
$\quad$ Other & 0.800 & 0.341 & 2.225 & {$[1.14,4.34]$} & 5.5 & 1 & 0.013 \\
\hline
\end{tabular}

Note. $\mathrm{CI}=$ confidence interval for odds ratio (OR). Reference category for Country=XKX. Reference category for Profession=Specialist Physician. $R^{2}=0.225$ (Nagelkerke). Model $\chi^{2}(10)=416.0, p<0.001,-2 \mathrm{LL}=2463.9$. 
Table S11 Illustrative steps needed to develop effective interventions using the MoVac/MovAd scales and the "Listen-Understand-Engage" framework [1]

\begin{tabular}{|c|c|c|}
\hline Step & Action & Example \\
\hline 1. Listen & $\begin{array}{l}\text { Survey Healthcare workers' sentiments towards vaccination } \\
\text { and/or vaccination advocacy in a given population using the } \\
\text { MoVac/MovAd tools [2]. }\end{array}$ & \\
\hline 2. Understand & $\begin{array}{l}\text { Identify the key barriers against vaccination and/or advocacy } \\
\text { through a Two-Step cluster profile of respondents. }\end{array}$ & $\begin{array}{l}\text { In the 2014-2015 survey reported in this study, the "Hesitant" } \\
\text { cluster was most hesitant about the value of flu vaccination. }\end{array}$ \\
\hline 3. Engage & $\begin{array}{l}\text { Identify, design, and implement an appropriate intervention } \\
\text { function (e.g., education, persuasion, incentivizing, coercion, } \\
\text { modelling; see [3]) }\end{array}$ & $\begin{array}{l}\text { Perception of importance may be influenced through } \\
\text { persuasion. For example, belief persistence is known to be } \\
\text { reduced if people can easily find counterarguments for their } \\
\text { beliefs [4]. Engaging hesitant HCWs to give a few key } \\
\text { reasons why flu vaccination might nevertheless be important } \\
\text { could work implicitly to increase their perception of the } \\
\text { importance of flu vaccination. }\end{array}$ \\
\hline
\end{tabular}

4. Listen

Carry out a post-test survey of HCWs' sentiments towards vaccination and/or vaccination advocacy and vaccination behavior rates
Post-intervention assessments are important to evaluate the impact of the interventions on staff engagement profile and to keep the cycle. A 'gold standard' for assessing interventions is a Solomon four-group design [5] which requires a randomized allocation to four groups: (1) pre-test intervention - post-test, (2) pre-test - post-test, (3) intervention - post-test, and (4) post-test.

[1] Thomson A, Watson M. Listen, Understand, Engage. Sci Transl Med 2012;4:138ed6-138ed6. doi:10.1126/scitranslmed.3004264.

[2] Vallée-Tourangeau G, Promberger M, Moon K, Wheelock A, Sirota M, Norton C, et al. Motors of influenza vaccination uptake and vaccination advocacy in healthcare workers: Development and validation of two short scales. Vaccine 2017. doi:10.1016/j.vaccine.2017.08.025.

[3] Michie S, Van Stralen MM, West R. The behaviour change wheel: a new method for characterising and designing behaviour change interventions. Implement Sci $2011 ; 6: 42$.

[4] Nestler S. Belief perseverance: The role of accessible content and accessibility experiences. Soc Psychol 2010;41:35-41. doi:10.1027/1864-9335/a000006.

[5] Kool VK, Agrawal R. On Using Experimental Designs. In: Hegde DS, editor. Essays Res. Methodol., New Delhi: Springer India; 2015, p. 169-83. doi:10.1007/978-81-322-2214-9 8. 